TRANSACTIONS OF THE

AMERICAN MATHEMATICAL SOCIETY

Volume 356, Number 12, Pages 4829-4838

S 0002-9947(04)03709-2

Article electronically published on June 25, 2004

\title{
AN ANALOGUE OF CONTINUED FRACTIONS IN NUMBER THEORY FOR NEVANLINNA THEORY
}

\author{
ZHUAN YE
}

\begin{abstract}
We show an analogue of continued fractions in approximation to irrational numbers by rationals for Nevanlinna theory. The analogue is a sequence of points in the complex plane which approaches a given finite set of points and at a given rate in the sense of Nevanlinna theory.
\end{abstract}

\section{INTRODUCTION}

Since P. Vojta 17 created a dictionary between Nevanlinna theory and Diophantine approximation theory, researchers (e.g. 7], [15, 20], 4] and [22]) from both fields have started to look for more analogues between these two theories. It is known that a famous theorem of Roth in number theory is analogous to a weak form of the second main theorem in Nevanlinna theory, and the Artin-Whaples product formula in number theory can be viewed as an analogue of the first main theorem in Nevanlinna theory. Theoretically speaking, we should be able to find an analogue of any theorem related to the Roth theorem in Diophantine approximation for Nevanlinna theory, and vice versa. An up-to-date account of these matters appears in [2], [18] and [14]. The author [23] has found an analogue of Khinchin's theorem for Nevanlinna theory, which has given an answer to one of S. Lang's questions in [9]. S. Lang also suggested (in a personal conversation) finding an analogue of the continued fractions for Nevanlinna theory. This is an interesting question that has been around for a while. In this paper, we find an analogue of continued fractions in approximation to irrational numbers by rationals for Nevanlinna theory. The analogue is a sequence of points in the complex plane which approaches a given finite set of points and at a given rate in the sense of Nevanlinna theory.

\section{NOTATION AND PRELIMINARIES}

For the convenience of the general readers, we briefly give some definitions and notation in Nevanlinna theory and continued fractions. Standard references are 3 and [12] for Nevanlinna theory, and [5] and [8] for continued fractions.

Let $f$ be a meromorphic function on the whole plane and $D_{r}=\{|z|<r\}$. Denote the number of poles of $f$ in $D_{r}$ by $n(f, \infty, r)$, and define $n(f, a, r)=$ $n(1 /(f-a), \infty, r)$ if $a \in \mathbb{C}$. We also let

$$
N(f, a, r)=\int_{0}^{r} \frac{n(f, a, t)-n(f, a, 0)}{t} d t+n(f, a, 0) \log r .
$$

Received by the editors July 25, 2002.

2000 Mathematics Subject Classification. Primary 30D35, 11J70.

Key words and phrases. Continued fraction, meromorphic function, approximation. 
We usually call $N(f, a, r)$ a counting function and simply write $N(f, \infty, r)$ as $N(f, r)$. This integrated function $N(f, a, r)$ occurs naturally in the main theorems of Nevanlinna theory. It measures the number of $a$-values in $D_{r}$.

The proximity function in Nevanlinna theory is defined by

$$
m(f, r)=\frac{1}{2 \pi} \int_{0}^{2 \pi} \log ^{+}\left|f\left(r e^{i \theta}\right)\right| d \theta
$$

where $\log ^{+} x=\max \{0, \log x\}, x \geq 0$; and $m(f, a, r)=m(1 /(f-a), r)$ for $a \in \mathbb{C}$. This function measures how close $f(z)$ is to the value $a$ in the sense of the average mean-value on $|z|=r$.

The characteristic function of $f$ in Nevanlinna theory is

$$
T(f, a, r)=N(f, a, r)+m(f, a, r) .
$$

However, by the first main theorem [3], we know that $T(f, a, r)$ is independent of $a$ up to a bounded constant, i.e.

$$
T(f, \infty, r)=T(f, a, r)+O(1) .
$$

In the sequel, we always write $T(f, r)=T(f, \infty, r)$.

Let $a_{1}, a_{2}, \cdots, a_{q}$ in $\mathbb{C} \cup\{\infty\}$ be $q(1 \leq q<\infty)$ distinct points. We define the error term of $f$ with respect to $\left\{a_{n}\right\}$ as

$$
S\left(f,\left\{a_{n}\right\}_{1}^{q}, r\right)=(q-2) T(f, r)-\sum_{j=1}^{q} N\left(f, a_{n}, r\right)+N_{R a m}(f, r),
$$

where $N_{\text {Ram }}(f, r)=N\left(f^{\prime}, 0, r\right)+2 N(f, \infty, r)-N\left(f^{\prime}, \infty, r\right)$. By the first main theorem, the error term can be written as

$$
S\left(f,\left\{a_{n}\right\}_{1}^{q}, r\right)=\sum_{j=1}^{q} m\left(f, a_{n}, r\right)+N_{R a m}(f, r)-2 T(f, r)+O(1) .
$$

According to Vojta's dictionary, the analogue of $\sum_{j=1}^{q} m\left(f, a_{n}, r\right)+N_{R a m}(f, r)$ for Diophantine approximation theory is $\alpha-p / q$, where $\alpha$ is an irrational number and $p / q$ is a rational number. Therefore, broadly speaking, the error term $S\left(f,\left\{a_{n}\right\}_{1}^{q}, r\right)$ measures how close $f\left(r e^{i \theta}\right)$ is to the points $\left\{a_{j}\right\}$ in the average mean-value on $|z|=r$ with a consideration of its ramification term.

In short, we say $S\left(f,\left\{a_{n}\right\}_{1}^{q}, r\right)$ measures the closeness of $f$ to $\left\{a_{n}\right\}_{1}^{q}$ in the sense of Nevanlinna theory. The coefficient 2 in (1), as P. Vojta and C. F. Osgood pointed out in [17] and 13], is the same as the power 2 in Roth's theorem. In general, the bigger $S\left(f,\left\{a_{n}\right\}_{1}^{q}, r\right)$ is, the better the approximation of $f\left(r e^{i \theta}\right)$ to the points $\left\{a_{n}\right\}$ is in the sense of Nevanlinna theory.

Let $v$ be a positive, increasing and continuous function with $\int_{1}^{\infty} d t /(t v(t))<\infty$. It is known (e.g. [1] and [2]) that, for any meromorphic function $f$ and any finite set of points $\left\{a_{j}\right\}_{1}^{q}$ in the extended complex plane,

$$
S\left(f,\left\{a_{j}\right\}_{1}^{q}, r\right) \leq \log (T(f, r) v(T(f, r)))+O(1)
$$

holds for all large $r$ outside a set of finite Lebesgue measure. This is the second main theorem in Nevanlinna theory with emphasis on the error term. Amazingly, S. Lang [6] conjectured the formation of the above inequality, which is a better inequality than the analogue of a well-known Roth theorem in number theory for Nevanlinna theory. In fact, the analogue of the above inequality for number theory is still an open question, which was raised by S. Lang in the 1960's. 
An expression of the form

$$
a_{0}+\frac{1}{a_{1}+\frac{1}{a_{2}+\cdots}}
$$

is called a continued fraction. In general, the letters $a_{0}, a_{1}, \cdots$ could be any independent variables. However, for the purposes of our paper, we always assume $a_{1}, a_{2}, \cdots$ to be positive integers and $a_{0}$ to be an arbitrary real number. For simplicity, we write the above infinite continued fraction in the form

$$
\left[a_{0} ; a_{1}, a_{2}, \cdots\right]
$$

and the finite continued fraction in the form

$$
\left[a_{0} ; a_{1}, a_{2}, \cdots, a_{n}\right] .
$$

For any real number $\alpha$, we denote by $a_{0}$ the largest integer not exceeding $\alpha_{0}$. If $\alpha$ is not an integer, then there is a real number $r_{1}>1$ such that $\alpha=a_{0}+1 / r_{1}$. Clearly, $\alpha=\left[a_{0} ; r_{1}\right]$. If $r_{1}$ is not an integer, we denote by $a_{1}$ the largest integer not exceeding $r_{1}$ and define the number $r_{2}>1$ by the equation $r_{1}=a_{1}+1 / r_{2}$. Note that $\alpha=\left[a_{0} ; a_{1}, r_{2}\right]$. The procedure can be continued as long as $r_{n}>1$ is not an integer. This method also gives us an algorithm for computing $a_{n}$.

If $\alpha$ is rational, the above process will stop after a finite number of steps. Therefore, there corresponds a unique finite continued fraction such that

$$
\left[a_{0} ; a_{1}, a_{2}, \cdots, a_{n}\right]=\alpha .
$$

Recall we always assume $a_{j}$ is a positive integer when $j \geq 1$ and the last element of every finite continued fraction must be different from unity.

If $\alpha$ is irrational, then there corresponds a unique infinite continued fraction $\left[a_{0} ; a_{1}, a_{2}, \cdots\right]$ with value equal to $\alpha$; i.e. set

$$
\frac{p_{n}}{q_{n}}=\left[a_{0} ; a_{1}, a_{2}, \cdots, a_{n}\right]
$$

where the fraction $p_{n} / q_{n}$ is irreducible and $q_{n}>0$; then $p_{n} / q_{n} \rightarrow \alpha$ as $n \rightarrow \infty$. The degree of accuracy of this approximation is, for all $n \geq 0$,

$$
\frac{1}{q_{n}\left(q_{n}+q_{n+1}\right)}<\left|\alpha-\frac{p_{n}}{q_{n}}\right| \leq \frac{1}{q_{n} q_{n+1}} .
$$

The inequality also implies that $\left|\alpha-p_{n} / q_{n}\right| \leq 1 / q_{n}^{2}$. The power 2 in this continued fraction approximation also corresponds to the coefficient 2 in (1), which is called by Khinchin the order of the approximation.

\section{StATEMENT OF RESUlts}

The theory of continued fractions tells us that, for any irrational number $\alpha$, there is a sequence of rational numbers $p_{n} / q_{n}$ such that $\lim _{n \rightarrow \infty} p_{n} / q_{n}=\alpha$. There are two advantages to the use of continued fractions in number theory. One is the control of its accuracy by using the inequalities (3), and the other is the existence of its algorithm, by which we can find $a_{j}$ 's, and then rational numbers $p_{n} / q_{n}$ as we see from the previous section.

On the other hand, Nevanlinna theory tells us that the error term of the second main theorem $S\left(f,\left\{a_{j}\right\}, r\right)$ in Nevanlinna theory reveals the degree of approximation of a meromorphic function $f$ to the finite set $\left\{a_{j}\right\}$ in the sense of the average mean-value on $|z|=r$. 
To help the reader understand the analogue of continued fractions for Nevanlinna theory, which is presented in the following Theorem, we would like to give a geometric interpretation of the analogue.

For any finite set of points $\left\{a_{n}\right\}_{1}^{q}$ in the extended complex plane, there is an infinite set of points $\left\{z_{j, p}\right\}_{j=1, p=0}^{\infty, p_{j}}$ in the complex plane, which is placed on a sequence of circles $\left\{|z|=r_{j}\right\}_{j=1}^{\infty}$ in a very special way so that the accuracy of approximation in the sense of Nevanlinna theory can be controlled at a given rate $\phi(r)$. The algorithm for finding the locations of those points $\left\{z_{j, p}\right\}_{j=1, p=0}^{\infty, p_{j}}$, which only depends on the function $\phi(r)$, can be seen in the proof of the Theorem. To verify the approximation of points $\left\{z_{j, p}\right\}_{j=1, p=0}^{\infty, p_{j}}$ to points $\left\{a_{n}\right\}_{1}^{q}$ at a given rate in the sense of Nevanlinna theory, we need a meromorphic function $f$ such that $S\left(f,\left\{a_{n}\right\}, r\right)$ is equal to the given rate.

To pursue the best possible theorem on the analogue of continued fractions for Nevanlinna theory, we have the following:

Theorem. Let $a_{1}, a_{2}, \cdots, a_{q}$ in $\mathbb{C} \cup\{\infty\}$ be $q(1 \leq q<\infty)$ distinct points. Let $p$ and $\phi$ be positive increasing differentiable functions with $\int_{1}^{\infty} d t / p(t)=\infty$ and $\int_{1}^{\infty} d t / t \phi(t)=\infty$, and define $\psi(r)=r \phi(r)$,

$$
P(r)=\int_{1}^{r} \frac{d t}{p(t)} \quad \text { and } \quad \Psi(r)=\int_{1}^{r} \frac{d t}{t \phi(t)} .
$$

Let $a_{1}, \cdots, a_{q}(1 \leq q<\infty)$ be any distinct points in the extended plane. If

$$
\limsup _{r \rightarrow \infty} \frac{p^{\prime}(r)}{\psi^{*}(r)}=A<\infty
$$

where $\psi^{*}(r)=\inf _{t \geq r} \psi^{\prime}(t)$, and there is a constant $C>1$ such that $\phi(3 r) \leq C \phi(r)$ for $r \geq 1$, then, for any $\tau^{\prime}>\tau>A$, there exists a meromorphic function $F$ such that

$$
\frac{1}{3} \Psi^{-1}(\tau P(r))+O(1) \leq T(F, r) \leq \Psi^{-1}\left(\tau^{\prime} P(r)\right)+O(1)
$$

holds for all sufficiently large $r$, where $\Psi^{-1}$ is the inverse of $\Psi$; and

$$
S\left(F,\left\{a_{j}\right\}, r\right)=\log \frac{T(F, r) \phi(T(F, r))}{p(r)}+O(1)
$$

holds for $r$ outside a set of finite Lebesgue measure.

Remark 1. In a conversation with S. Lang in the 90's, he told me that he had an idea about an analogue of continued fractions for function theory in the 60 's. It will be great if this is the same as he had at that time.

Remark 2. The Theorem complements some results in [11, 4], [1], [19] and [21]. The Theorem also gives a solution to the inverse problem of the error term.

Remark 3. The function $p$ is introduced in order to adjust the growth of the function $f$ and the growth of the error term $S\left(f,\left\{a_{j}\right\}, r\right)$. Moreover, the condition $\int d r / \psi(r)=\infty$ is necessary if $p(r)=1$, as we can see from (2).

Remark 4. Statement (i) of the Theorem is about the control of the growth of the function $f$. Statement (ii) is about the accuracy of $f$ 's approximation to points $\left\{a_{n}\right\}_{1}^{q}$ on $|z|=r$ in the sense of Nevanlinna theory, which is a counterpart of the inequalities (3). 
Remark 5. The proof of the Theorem also gives an algorithm for finding the function $F$ which is a product of $\left\{z-z_{j, p}\right\}_{j=1, p=0}^{\infty, p_{j}}$. Broadly speaking, the partial product is a counterpart of $p_{n} / q_{n}$ in continued fractions.

Remark 6. If one of the $a_{n}$ 's, say $a_{1}$, is infinity, then the function $F$ in the Theorem is an entire function. Moreover, the function $F$ is independent of $\left\{a_{n}\right\}_{n=2}^{q}$, i.e., when $r$ is outside a set of finite Lebesgue measure,

$$
S\left(F,\{\infty\} \cup\left\{a_{n}\right\}_{n=2}^{q}, r\right)=\log \frac{T(F, r) \phi(T(F, r))}{p(r)}+O(1)
$$

holds for any distinct complex numbers $a_{2}, \cdots, a_{q}$ in $\mathbb{C}$.

Remark 7. If one fixes the approximation data $p(r)$ and $\phi(r)$, all functions produced in the Theorem are related to each other by linear fractional transformations depending on $\left\{a_{j}\right\}$. So, the sharpness of the estimate is from the ramification term $N_{\text {Ram }}$ which has no known counterpart in number theory. It would be interesting if one can construct a function having $N_{\text {Ram }}(r)=0$ as well as the estimates in our Theorem.

\section{Proof of the Theorem}

Proof of the Theorem. Let $\tau^{\prime}>\tau>A$. Define

$$
\Omega(r)=\Psi^{-1}(\tau P(r)) \text { and } \omega(r)=r \Omega^{\prime}(r) .
$$

Equation (4) implies that, for any $\delta>0$,

$$
P(r) \geq \frac{\Psi(r)}{A+\delta},
$$

for all sufficiently large $r$. Let $1<\alpha<\tau / A$; noting the fact that $\Psi^{-1}(s r) \geq$ $\left(\Psi^{-1}(r)\right)^{s}(s \geq 1$ and $r>0)$, we have, for sufficiently large $r$,

$$
\Omega(r) \geq \Psi^{-1}\left(\frac{\tau \Psi(r)}{A+\delta}\right) \geq r^{\tau /(A+\delta)} \geq r^{\alpha},
$$

where $\delta$ is a small positive number such that $\alpha<\tau /(A+\delta)<\tau / A$. Equation (4) and the fact $\psi(r) \rightarrow \infty$ show that

$$
\frac{\psi(r)}{p(r)} \geq \frac{1}{A+\delta}
$$

for all sufficiently large $r$. Consequently, by a computation of $\Omega^{\prime}(r)$ and (5),

$$
\omega(r)=\tau r \frac{\psi(\Omega(r))}{p(r)} \geq \tau r r^{\alpha} \frac{\phi\left(r^{\alpha}\right)}{p(r)} \geq \tau r^{\alpha} \frac{\psi(r)}{p(r)} \geq \frac{\tau}{A+\delta} r^{\alpha} \geq r^{\alpha},
$$

for sufficiently large $r$. Moreover, we have

$$
\omega^{\prime}(r)=\frac{\tau^{2} r \psi(\Omega(r))}{p(r)^{2}}\left(\frac{p(r)}{\tau r}+\psi^{\prime}(\Omega(r))-\frac{p^{\prime}(r)}{\tau}\right) .
$$

It follows from (5) and (44) that

$$
\limsup _{r \rightarrow \infty} \frac{p^{\prime}(r)}{\psi^{\prime}(\Omega(r))} \leq \limsup _{r \rightarrow \infty} \frac{p^{\prime}(r)}{\psi^{\prime}(r)} \leq \limsup _{r \rightarrow \infty} \frac{p^{\prime}(r)}{\psi^{*}(r)}=A .
$$

Thus, $\omega^{\prime}(r)>0$ for sufficiently large $r$, since $\tau>A$. Therefore $\omega$ is strictly increasing when $r$ is sufficiently large. 
Let $\left\{r_{j}\right\}$ be the sequence defined by

$$
\omega\left(r_{j}\right)=2^{j+1}, \quad \text { for } j=1,2,3, \cdots .
$$

There exists an integer $j_{0}$ such that the sequence $\left\{r_{j}\right\}_{j_{0}}^{\infty}$ is uniquely determined, strictly increasing and unbounded, since $\omega(r)$ increases to infinity. Without loss of generality, we assume that $r_{j_{0}} \geq 1$. Furthermore, inequality (6) gives, for all large $j$, say, $j \geq j_{0}$,

$$
r_{j} \leq 2^{(j+1) / \alpha} \text { and } 2^{j(1-1 / \alpha)} \geq 4 j^{3} .
$$

Define

$$
f(z)=\prod_{j=j_{0}}^{\infty}\left(1-\left(\frac{z}{r_{j}}\right)^{2^{j}}\right) .
$$

It turns out that

$$
\frac{1}{2} \omega(r)-2^{j_{0}} \leq n(f, 0, r) \leq \omega(r)-2^{j_{0}}, \quad r \geq r_{j_{0}} .
$$

For $j \geq j_{0}$, define

$$
s_{j}=r_{j}-\frac{1}{j^{2}}, \quad S_{j}=r_{j}+\frac{1}{j^{2}}, \text { and } E=\bigcup_{j \geq j_{0}}\left[s_{j}, S_{j}\right] .
$$

Obviously, $E$ is of finite Lebesgue measure. For a point $z$ such that $|z|=r \notin E$ and $|z|>r_{0}=r_{j_{0}}$, let $k$ be the unique integer such that $S_{k}<|z|<s_{k+1}$. Then we obtain from (7) that

$$
2^{j} \log \left|\frac{z}{r_{j}}\right| \geq 2^{j} \log \left(1+\frac{1}{j^{2} r_{j}}\right) \geq \frac{2^{j-1}}{j^{2} r_{j}} \geq j, \quad j_{0} \leq j \leq k,
$$

and

$$
2^{j} \log \left|\frac{z}{r_{j}}\right| \leq 2^{j} \log \left(1-\frac{1}{j^{2} r_{j}}\right) \leq \frac{-2^{j}}{j^{2} r_{j}} \leq-j, \quad j>k .
$$

It follows from (10) and (11) that $f$ is entire and that, for the above $z$ and $k$,

$$
\begin{aligned}
\log |f(z)| & \geq \sum_{j=j_{0}}^{k} \log \left(\left|\frac{z}{r_{j}}\right|^{2^{j}}-1\right)+\sum_{j=k+1}^{\infty} \log \left(1-\left|\frac{z}{r_{j}}\right|^{j^{j}}\right) \\
& \geq \sum_{j=j_{0}}^{k} \log \left(2^{-1}\left|\frac{z}{r_{j}}\right|^{2^{j}}\right)-\sum_{j=k+1}^{\infty}\left|\frac{z}{r_{j}}\right|^{2^{j}} \\
& \geq-\left(k-j_{0}+1\right) \log 2+\sum_{j=j_{0}}^{k} j-\sum_{j=k+1}^{\infty} e^{-j} \\
& =B_{k} .
\end{aligned}
$$

As $|z|=r$ goes to infinity, $k$ goes to infinity and, thus, $B_{k}$ tends to infinity. Therefore, $f(z) \rightarrow \infty$ when $z \rightarrow \infty$ and $|z|=r \notin E$; consequently,

$$
m(f, 0, r)=0 \text {, }
$$


for sufficiently large $r \notin E$. Thus, we have from (9) and the fact $f(0)=1$ that, for sufficiently large $r \notin E$,

$$
\begin{aligned}
T(f, r) & =T(f, 0, r)+\log |f(0)|=N(f, 0, r)=\int_{r_{0}}^{r} \frac{n(f, 0, t)}{t} d t \leq \int_{r_{0}}^{r} \frac{\omega(t)}{t} d t \\
& =\int_{r_{0}}^{r} \Omega^{\prime}(t) d t \leq \Omega(r)=\Psi^{-1}(\tau P(r)) .
\end{aligned}
$$

Let $\lambda=\left(\tau^{\prime} / \tau\right)^{1 / 2}>1$. Since $E$ has a finite linear measure, for any sufficiently large $r$ ( $r$ may be in the set $E$ ), there is an $r^{\prime}$ such that $r \leq r^{\prime} \leq \lambda r$ and $r^{\prime} \notin E$. So, by (12) and the fact that $P(\lambda r) \leq \lambda P(r)+P(\lambda)$ for $r>1$,

$$
\begin{aligned}
T(f, r) & \leq T\left(f, r^{\prime}\right) \leq \Psi^{-1}\left(\tau P\left(r^{\prime}\right)\right) \leq \Psi^{-1}(\tau P(\lambda r)) \\
& \leq \Psi^{-1}(\tau(\lambda P(r)+P(\lambda))) \leq \Psi^{-1}\left(\tau^{\prime} P(r)\right) .
\end{aligned}
$$

On the other hand, using (9), for all $r \geq r_{0}$ without exception, we have

(14) $T(f, r) \geq N(f, 0, r) \geq \frac{1}{2}\left(\Omega(r)-\Omega\left(r_{0}\right)\right)-2^{j_{0}} \log r>\frac{1}{3} \Psi^{-1}(\tau P(r))=\frac{1}{3} \Omega(r)$.

For sufficiently large $r \notin E$, by (12), we obtain

$$
\psi(\Omega(r))=\Omega(r) \phi(\Omega(r)) \geq T(f, r) \phi(T(f, r)) ;
$$

and by (14), noting $\phi(3 r) \leq C \phi(r)$, we get

$$
\psi(\Omega(r)) \leq 3 T(f, r) \phi(3 T(f, r)) \leq 3 C T(f, r) \phi(T(f, r)) .
$$

It follows that, for sufficiently large $r \notin E$,

$$
\log \frac{\omega(r)}{r}=\log \frac{\tau \psi(\Omega(r))}{p(r)}=\log \frac{T(f, r) \phi(T(f, r))}{p(r)}+O(1) .
$$

For a point $z$ such that $|z|=r \notin E$ and $|z| \geq r_{0}$,

$$
z \frac{f^{\prime}(z)}{f(z)}=\sum_{j=j_{0}}^{k} 2^{j}+\sum_{j=j_{0}}^{k} \frac{2^{j}}{\left(z / r_{j}\right)^{2^{j}}-1}+\sum_{j=k+1}^{\infty} \frac{2^{j}\left(z / r_{j}\right)^{j^{j}}}{\left(z / r_{j}\right)^{2^{j}}-1},
$$

where $k$ is defined as above. By (10) and (11), we obtain

$$
\begin{aligned}
&\left|\sum_{j=k+1}^{\infty} \frac{2^{j}\left(z / r_{j}\right)^{2^{j}}}{\left(z / r_{j}\right)^{2^{j}}-1}\right| \leq \sum_{j=k+1}^{\infty} 2^{j+1} e^{-j}=o(1), \\
&\left|\sum_{j=j_{0}}^{k} \frac{2^{j}}{\left(z / r_{j}\right)^{2 j}-1}\right| \leq \sum_{j=j_{0}}^{k} 2^{j+1} e^{-j}=O(1) .
\end{aligned}
$$

Hence, noting that $\sum_{j=j_{0}}^{k} 2^{j}=n(f, 0, r)$ for $r \geq r_{0}$ and $r \notin E$, we have

$$
\left|\frac{f^{\prime}}{f}\left(r e^{i \theta}\right)\right|=\frac{n(f, 0, r)}{r}(1+o(1))
$$

for $r \notin E$. Consequently, (9) implies that

$$
\frac{1}{2 \pi} \int_{0}^{2 \pi} \log \left|\frac{f^{\prime}}{f}\left(r e^{i \theta}\right)\right| d \theta=\log \frac{n(f, 0, r)}{r}+O(1)=\log \frac{\omega(r)}{r}+O(1)
$$

holds for $r \notin E$.

Now we consider two cases.

Case 1: If $\infty \in\left\{a_{n}\right\}_{j}^{q}$, without loss of generality, we assume that $a_{1}=\infty$. 
Let $F(z)=f(z)$, where $f$ is defined as in (8). It follows from (13) and (14) that (i) is proved. Let $\|a, b\|$ be the spherical distance, and

$$
\Gamma_{F}=\gamma_{F} \prod_{j=1}^{q} \frac{1}{\left\|F, a_{j}\right\|^{2}}=\frac{\left|F^{\prime}\right|^{2}}{\left(1+|F|^{2}\right)} \prod_{j=2}^{q} \frac{1}{\left\|F, a_{j}\right\|^{2}},
$$

where $\gamma_{F}=\left(F^{\#}\right)^{2}=\left(|F| /\left(1+|F|^{2}\right)\right)^{2}$. It follows that

$$
\log \Gamma_{F}\left(r e^{i \theta}\right)=2 \log \left|\frac{F^{\prime}}{F}\left(r e^{i \theta}\right)\right|+O(1)=2 \log \left|\frac{f^{\prime}}{f}\left(r e^{i \theta}\right)\right|+O(1)
$$

as $r \rightarrow \infty$ outside $E$, since $F(z)=f(z) \rightarrow \infty$ as $z \rightarrow \infty$ and $|z|=r \notin E$. Using the Green-Jensen formula [2, Theorem 1.10.3 (pg. 34)] and (15), we get

$$
\begin{aligned}
S\left(F,\left\{a_{j}\right\}, r\right) & =\frac{1}{4 \pi} \int_{0}^{2 \pi} \log \Gamma_{F}\left(r e^{i \theta}\right) d \theta+O(1) \\
& =\frac{1}{2 \pi} \int_{0}^{2 \pi} \log \left|\frac{f^{\prime}}{f}\left(r e^{i \theta}\right)\right| d \theta+O(1)=\log \frac{\omega(r)}{r}+O(1) \\
& =\log \frac{T(F, r) \phi(T(F, r))}{p(r)}+O(1) .
\end{aligned}
$$

Case 2: If $\infty \notin\left\{a_{n}\right\}_{j}^{q}$, we take $F(z)=a_{1}+1 / f(z)$, where again $f$ is defined as in (8). By the first main theorem, $T(F, r)=T(f, r)+O(1)$. So (i) is proved in this case by using (13) and (14). Moreover,

$$
\Gamma_{F}=\gamma_{F} \prod_{j=1}^{q} \frac{1}{\left\|F, a_{j}\right\|^{2}}=\left(\frac{\left|f^{\prime}\right|}{|f|}\right)^{2} \frac{1+\left|a_{1}\right|^{2}}{\left(1+|F|^{2}\right)} \prod_{j=2}^{q} \frac{1}{\left\|F, a_{j}\right\|^{2}} .
$$

Noting, for sufficiently large $r \rightarrow \infty$ outside $E$, that $f(z) \rightarrow \infty$, we have

$$
\log \Gamma_{F}\left(r e^{i \theta}\right)=2 \log \left|\frac{f^{\prime}}{f}\left(r e^{i \theta}\right)\right|+O(1) .
$$

By following the steps used in Case 1, we obtain the Theorem.

\section{EXAMPLES}

As S. Lang and H. Trotter in [10] and [8] have given some computational information for the behavior of certain algebraic numbers with respect to approximation by their continued fractions, we would like to give two examples to illustrate the accuracy and the algorithm of our approximation in the sense of Nevanlinna theory. Example 1 shows a "good" algorithm and "bad" accuracy, while Example 2 shows a "bad" algorithm and "good" accuracy. The reader can find other examples about the error terms of classical functions in [16] and [2].

Example 1. Let $p(r)=r$ and $\phi(r)=1$, and let $a_{1}=\infty$ and $a_{2}, a_{3}, \cdots, a_{q}$ be any distinct complex numbers in $\mathbb{C}$. Thus we have

$$
P(r)=\ln r, \quad \Psi(r)=\ln r, \quad \text { and } \quad A=1 .
$$

By taking $\tau=2>A$, we obtain from the proof of our Theorem that

$$
\Omega(r)=r^{2} \quad \text { and } \quad \omega(r)=2 r^{2} .
$$


Thus the sequence of circles, on which the sequence of points $\left\{z_{j, p}\right\}$ are placed, is $\left\{|z|=2^{j / 2}\right\}_{j=1}^{\infty}$. Recall the $r_{j}$ is defined by the equation $\omega\left(r_{j}\right)=2^{j+1}$. In order to compare this example with the next example, we list the first twenty $r_{j}$ 's:

$$
\begin{array}{llll}
r_{1}=1.414213562 & r_{2}=2.000000000 & r_{3}=2.828427125 & r_{4}=4.000000000 \\
r_{5}=5.656854249 & r_{6}=8.000000000 & r_{7}=11.31370850 & r_{8}=16.00000000 \\
r_{9}=22.62741700 & r_{10}=32.00000000 & r_{11}=45.25483400 & r_{12}=64.00000000 \\
r_{13}=90.50966799 & r_{14}=128.0000000 & r_{15}=181.0193360 & r_{16}=256.0000000 \\
r_{17}=362.0386720 & r_{18}=512.0000000 & r_{19}=724.0773439 & r_{20}=1024.000000
\end{array}
$$

Furthermore, for any fixed positive integer $j$,

$$
z_{j, p}=2^{j / 2} \omega_{j}^{p}, \quad p=0,1,2, \cdots, 2^{j}-1,
$$

where $\omega_{j}=e^{i\left(2 \pi / 2^{j}\right)}$ is a $2^{j}$-th root of unity. The entire function determined by the sequence $\left\{z_{j, p}\right\}_{j=1, p=0}^{\infty, 2^{j}-1}$ is

$$
F(z)=\prod_{j=1}^{\infty}\left(1-\left(\frac{z}{2^{j / 2}}\right)^{2^{j}}\right)
$$

which has the property, for all large $r$ outside a set of finite Lebesgue measure,

$$
S\left(F,\left\{a_{n}\right\}_{1}^{q}, r\right)=\log T(F, r)+O(1) .
$$

Theoretically speaking, the smaller $p$ and bigger $\phi$ are, the better the approximation is since the error term $S\left(f,\left\{a_{n}\right\}_{1}^{q}, r\right)$ gets bigger.

Example 2. Let $p(r)=1$ and $\phi(r)=\log (10 r)$, and let $a_{1}=\infty$ and $a_{2}, a_{3}, \cdots, a_{q}$ be any distinct complex numbers in $\mathbb{C}$. Thus we have

$$
P(r)=r-1, \quad \Psi(r)=\log \log (10 r), \quad \text { and } \quad A=0 .
$$

By taking $\tau=1>A$, we obtain from the proof of our Theorem that

$$
\Omega(r)=10^{10^{r-1}-1} \quad \text { and } \quad \omega(r)=(\ln 10)^{2} r 10^{r-1} 10^{10^{r-1}-1} .
$$

As we know, the sequence of circles $\left\{|z|=r_{j}\right\}$ is determined by the equation $\omega\left(r_{j}\right)=2^{j+1}$. We could compute these $r_{j}$ 's by using computers. Here we list the first twenty $r_{j}$ 's:

$$
\begin{array}{llll}
r_{1}=.966545620 & r_{2}=1.04635090 & r_{3}=1.11823735 & r_{4}=1.18313965 \\
r_{5}=1.24196365 & r_{6}=1.29552487 & r_{7}=1.34453111 & r_{8}=1.38958558 \\
r_{9}=1.43119816 & r_{10}=1.46979874 & r_{11}=1.50575017 & r_{12}=1.53935954 \\
r_{13}=1.57088784 & r_{14}=1.60055791 & r_{15}=1.62856093 & r_{16}=1.65506180 \\
r_{17}=1.68020341 & r_{18}=1.70411022 & r_{19}=1.72689115 & r_{20}=1.74864191
\end{array}
$$

Again, the entire function $F$ is defined as in (8) and the zeros of the function $F$ are $z_{j, p}=r_{j} \omega_{j}^{p}$, where $\omega_{j}$ is defined as in Example 1, and the function $F$ satisfies, for all large $r$ outside a set of finite Lebesgue measure,

$$
S\left(F,\left\{a_{n}\right\}_{1}^{q}, r\right)=\log T(F, r) \log \log (10 T(F, r))+O(1) .
$$

\section{ACKNOWLEDGments}

I would like to thank Serge Lang for raising the question and for his enthusiasm, David Rusin for showing a computing software and the referee for helpful suggestions such as Remark 7. 


\section{REFERENCES}

1. H. Chen and Z. Ye, The error term in Nevanlinna's inequality, Science in China (Series A), 43, (2000), 1060-1066. MR 1802149 (2001k:30039)

2. W. Cherry and Z. Ye, Nevanlinna's Theory of Value Distribution, Springer-Verlag, Monographs in Math., 2001. MR 1831783 (2002h:30030)

3. W. Hayman, Meromorphic Functions, Clarendon Press, 1964. MR 0164038 (29:1337)

4. A. Hinkkanen, A sharp form of Nevanlinna's second fundamental theorem, Invent. Math., 108, (1992), 549-574. MR 1163238 (93c:30045)

5. A. Ya. Khinchin, Continued Fractions, Chicago University Press, 1964. MR 0161833 (28:5037)

6. S. Lang, The error term in Nevanlinna theory, Duke Math. J., 56, (1988), 193-218. MR 0932862 (89g:32037)

7. S. Lang, The error term in Nevanlinna theory, Bull. of the AMS, 22, (1990), 115-125. MR 1003864 (90k:32080)

8. S. Lang, Introduction to Diophantine Approximations, Springer-Verlag, 1995. MR 1348400 (96h:11067)

9. S. Lang and W. Cherry, Topics in Nevanlinna Theory, Springer-Verlag, Lecture Notes in Math., 1433, 1990. MR 1069755 (91k:32025)

10. S. Lang and H. Trotter, Continued fractions of some algebraic numbers, J. reine u. angew. Math., 255, (1972), 112-134. MR 0306131 (46:5258)

11. J. Miles, A sharp form of the lemma on the logarithmic derivative, J. London Math. Soc., 45, (1992), 243-254. MR 1171552 (93i:30026)

12. R. Nevanlinna, Analytic Functions, Springer, 1970. MR 0279280 (43:5003)

13. C. F. Osgood, Sometimes effective Thue-Siegel-Roth-Schimidt Nevanlinna bounds or better, J. Number Theory, 21, (1985), 347-398. MR 0814011 (87f:11046)

14. M. Ru, Nevanlinna Theory and its Relation to Diophantine Approximation, World Scientific Publishing Co. Inc., 2001. MR 1850002 (2002g:11106)

15. M. Ru and P. Vojta, Schmidt's subspace theorem with moving targets, Invent. Math., 127, (1997), 51-65. MR 1423025 (97g:11076)

16. L. R. Sons and Z. Ye, The best error terms of classical functions, Complex Variables, 28, (1995), 55-66. MR 1700264 (2000f:30020)

17. P. Vojta, Diophantine Approximations and Value Distribution Theory, Springer-Verlag, Lecture Notes in Math., 1239, 1987. MR 0883451 (91k:11049)

18. P. Vojta, Nevanlinna theory and Diophantine approximation, Math. Sci. Res. Inst. Publ., 37, (1999), 535-564. MR 1748613 (2001j:11072)

19. Y. Wang, Sharp forms of Nevanlinna's error terms, J. Anal. Math., 71, (1997), 87-102. MR 1454245 (98f:30033)

20. P. M. Wong and W. Stoll, Second main theorem of Nevanlinna theory for non-equidimensional meromorphic maps, Amer. J. Math., 116, (1994), 1031-1071. MR 1296724 (95g:32042)

21. Z. Ye, On Nevanlinna's error terms, Duke Math. J., 64, (1991), 243-260. MR 1136375 (93a:30039)

22. Z. Ye, On Nevanlinna's second main theorem in projective space, Invent. Math., 122, (1995), 475-507. MR 1359601 (96j:32030)

23. Z. Ye, An analogue of Khinchin's theorem in Diophantine approximation for Nevanlinna theory, XVII Rolf Nevanlinna Colloquium; Walter de Gruyter and Company, (1996), 309319. MR 1427096 (97h:30046)

Department of Mathematical Sciences, Northern Illinois University, DeKalb, IlliNOIS 60115

E-mail address: ye@math.niu.edu 